OPEN ACCESS

Edited by:

Anders Fasth,

University of Gothenburg, Sweden

Reviewed by:

Victoria Bordon,

Ghent University, Belgium

Fabio Candotti,

Centre Hospitalier Universitaire

Vaudois (CHUV), Switzerland

${ }^{*}$ Correspondence:

Hirokazu Kanegane

hkanegane.ped@tmd.ac.jp

${ }^{\dagger}$ These authors have contributed equally to this work

Specialty section:

This article was submitted to Primary Immunodeficiencies,

a section of the journal

Frontiers in Immunology

Received: 30 September 2021

Accepted: 28 January 2022

Published: 23 February 2022

Citation:

Tanita K, Kawamura Y, Miura H, Mitsuiki N, Tomoda T, Inoue $K$, Iguchi $A$, Yamada $M$, Yoshida T, Muramatsu H, Tada N,

Matsui T, Kato M, Eguchi K, Ishimura M, Ohga S, Imai K,

Morio T, Yoshikawa T and Kanegane H

(2022) Case Report: Rotavirus

Vaccination and Severe Combined

Immunodeficiency in Japan.

Front. Immunol. 13:786375

doi: 10.3389/fimmu.2022.786375

\title{
Case Report: Rotavirus Vaccination and Severe Combined Immunodeficiency in Japan
}

\begin{abstract}
Kay Tanita ${ }^{1 \dagger}$, Yoshiki Kawamura ${ }^{2 \dagger}$, Hiroki Miura ${ }^{2}$, Noriko Mitsuiki ${ }^{1}$, Takahiro Tomoda ${ }^{1}$, Kento Inoue ${ }^{1}$, Akihiro Iguchi ${ }^{3,4}$, Masafumi Yamada ${ }^{3}$, Taro Yoshida ${ }^{5}$, Hideki Muramatsu ${ }^{5}$, Norimasa Tada ${ }^{6}$, Toshihiro Matsui ${ }^{4}$, Motohiro Kato $^{4}$, Katsuhide Eguchi ${ }^{7}$, Masataka Ishimura ${ }^{7}$, Shouichi Ohga ${ }^{7}$, Kohsuke Imai $^{8}$, Tomohiro Morio ${ }^{1}$, Tetsushi Yoshikawa ${ }^{2}$ and Hirokazu Kanegane ${ }^{9 *}$

1 Department of Pediatrics and Developmental Biology, Graduate School of Medical Sciences, Tokyo Medical and Dental University (TMDU), Tokyo, Japan, 2 Department of Pediatrics, Fujita Health University School of Medicine, Toyoake, Japan, ${ }^{3}$ Department of Pediatrics, Faculty of Medicine and Graduate School of Medicine, Hokkaido University, Sapporo, Japan, ${ }^{4}$ Children's Cancer Center, National Center for Child Health and Development, Tokyo, Japan, ${ }^{5}$ Department of Pediatrics, Nagoya University Graduate School of Medicine, Nagoya, Japan, ${ }^{6}$ Department of Pediatrics, Tsuchiura Kyodo General Hospital, Ibaraki, Japan, ${ }^{7}$ Department of Pediatrics, Graduate School of Medical Sciences, Kyushu University, Fukuoka, Japan, ${ }^{8}$ Department of Community Pediatrics, Perinatal and Maternal Medicine, Graduate School of Medical and Dental Sciences, Tokyo Medical and Dental University (TMDU), Tokyo, Japan, ${ }^{9}$ Department of Child Health and Development, Graduate School of Medical and Dental Sciences, Tokyo Medical and Dental University (TMDU), Tokyo, Japan
\end{abstract}

Severe combined immunodeficiency (SCID) is an inborn error of immunity that occurs in approximately 1 in 50,000 births, mainly due to impaired lymphocyte differentiation. Without curative treatment, such as hematopoietic cell transplantation (HCT) or gene therapy, severe infection in the first year of life could make this condition fatal. The results of HCT are poor when patients have active infections, thus requiring early diagnosis before onset of infection. In five cases of SCID diagnosed in Japan, the oral rotavirus vaccine had been administered before diagnosis. In this study, we demonstrated that the rotavirus from their stools was a vaccine-derived strain. In some cases, severe gastroenteritis triggered the diagnosis of SCID. However, newborn screening for SCID is available before the first rotavirus vaccination using assays for the detection of T-cell receptor excision circles (TRECs). Therefore, to improve the prognosis of patients with SCID in Japan, we should establish a screening system of TRECs for newborns throughout Japan.

Keywords: severe combined immunodeficiency, hematopoietic cell transplantation, rotavirus, vaccination, T-cell receptor excision circles (TREC)

\section{INTRODUCTION}

Severe combined immunodeficiency (SCID) is a critical inborn error of immunity (IEI) that causes cellular and humoral immunity failure due to impaired lymphocyte differentiation, resulting in severe infections from infancy. Therefore, hematopoietic cell transplantation (HCT) or gene therapy is required as a curative therapy.

Rotavirus infection is a gastrointestinal infection that occurs in infants and children. Although mild cases can resolve spontaneously, some cases can cause fatal dehydration and encephalitis or 
encephalopathy, leading to the hospitalization of many patients. Although live vaccination is contraindicated (1), patients with SCID often remain asymptomatic until early infancy and are rarely diagnosed before 2 months of age when oral rotavirus vaccination is initiated. Furthermore, Rotarix ${ }^{\circledR}$, a live, monovalent, attenuated, human rotavirus vaccine (RV1), and RotaTeq ${ }^{\circledR}$, a live, pentavalent, human-bovine reassortant rotavirus vaccine (RV5), were initiated as arbitrary vaccination in 2011 and 2012, respectively, in Japan. Regular administration of these vaccines was initiated in October 2020. This initiative was taken after realizing that the number of cases vaccinated before the diagnosis of SCID will increase.

Several severe cases of rotavirus gastroenteritis caused by vaccine strains in SCID have been reported overseas $(2,3)$ as well as in Japan $(4,5)$. This report summarizes the cases of five patients with SCID who were vaccinated before being diagnosed with SCID in Japan.

\section{MATERIALS AND METHODS}

\section{RNA Extraction from Patient Stool and Serum}

Ten percent suspensions $(1 \mathrm{ml})$ of each stool sample were prepared in physiological saline solution or swab samples were rinsed in $500 \mu \mathrm{l}$ of physiological saline solution. Each suspension was then centrifuged for $20 \mathrm{~min}$ at $4,000 \times g$ and $140 \mu \mathrm{l}$ of the supernatant was used for RNA extraction. Finally, viral doublestranded RNAs (dsRNAs) were extracted from the stool suspension and $140-\mu \mathrm{l}$ sera using a QIAamp Viral RNA Mini Kit (Qiagen, Hilden, Germany).

\section{Sanger Sequencing}

First, RNA was extracted from patients' stool samples and RotaTeq and complementary DNA was synthesized using the High-Capacity cDNA Reverse Transcription Kit (Foster City, CA, USA). DNA was amplified by polymerase chain reaction (PCR) using primers for human rotavirus A gene 10, which encodes the rotavirus Non-structural protein 4 (NSP4) gene, and sequenced using the Sanger method (forward primer: 5'GGGCTTTTAAAAGTTCTGTTCCGAG-3', reverse primer: 5'GGTCACACTAAGACCATTCC-3’). Finally, we compared nucleotide and amino acid sequences of rotavirus strains derived from patients' stool samples, the RotaTeq WC3 strain, and the wild-type human/Wa strain.

\section{RNA Extraction and RV1- and RV5-Specific Real-Time Reverse Transcription-Polymerase Chain Reaction}

The stool samples were analyzed by real-time reverse transcriptase-polymerase chain reaction (RT-PCR) for the presence of RV5, RV1, and wild-type strains (6), and details about the real-time RT-PCR analysis were described elsewhere (7). Real-time RT-PCR was performed on a Fast Optical 48-Well Reaction Plate using a TaqMan RNA-to-Ct 1-Step kit (Thermo Fisher Scientific, Waltham, MA). In addition, single-well denaturation, reverse transcription, and amplification were performed on a StepOne Real-Time PCR system in standard mode (Thermo Fisher Scientific). Thermocycling conditions included a 15 -min hold at $48^{\circ} \mathrm{C}$, a 10 -min cycle at $95^{\circ} \mathrm{C}$, and 45 cycles of $15 \mathrm{~s}$ at $95^{\circ} \mathrm{C}$ and $1 \mathrm{~min}$ at $60^{\circ} \mathrm{C}$. Each rotavirus vaccine strain-specific real-time RT-PCR amplified the designated vaccine strain only, and no cross-reaction was observed with any of the wild-type strains. Meanwhile, realtime RT-PCR to detect Non-structural protein 3 (NSP3) gene in wild-type strains could also detect the two vaccine strains. Serially diluted purified rotavirus virions were used to determine the lower detection limits of RV5 (10 copies/ reaction), RV1 (50 copies/reaction), and wild type (50 copies/ reaction) using real-time RT-PCR. While the RNA extracted from RV5 and RV1 was used as a positive control for vaccine virus strains, the KU strain (G1P [8]) was used as a positive control for the wild-type virus.

\section{RESULTS}

\section{Case Reports}

Cases were collected throughout Japan from 2015 to 2020 (Figure 1 and Table 1). The first case (P1) was that of a patient with X-linked SCID, interleukin (IL)-2 receptor gamma (CD132) deficiency, who received the first dose of RV5 at 8 weeks of age (5, 8 ). He developed respiratory syncytial virus infection and interstitial pneumonia at 5 months of age and was admitted to the hospital. Upon recovery after a 3-week treatment, he had splenomegaly and hypergammaglobulinemia (immunoglobulin $\mathrm{G}(\mathrm{IgG})>3,000 \mathrm{mg} / \mathrm{dl})$. Further examination revealed natural killer (NK)-cell deficiency and skewing to memory $\mathrm{CD}^{+} \mathrm{T}$ cells, and IEI was suspected. Genetic analysis revealed the causative agent to be the IL2RG variant (c.676C>T, p.R226C). However, while preparing for HCT, the patient experienced loose stools, and a rapid test showed that he was rotavirus-positive. Cord blood transplantation (CBT) was performed at 11 months of age at Tokyo Medical and Dental University. The patient presented with loose stools from day 0 to day 11 after CBT. Rotavirus antigen test turned negative on day 28 .

The second case (P2), also with X-SCID, was administered the first dose of RV1 at 9 weeks of age. He developed severe Pneumocystis pneumonia (PCP) at 4 months, requiring mechanical ventilation. Flow cytometry revealed NK-cell deficiency with maternal $\mathrm{T}$ cells and lack of CD132 on lymphocytes, and the patient was diagnosed with X-SCID with the IL $2 R G$ variant (c.202 G>T, p.E68*). Although gastrointestinal symptoms were not the main symptoms, the patient tested positive for the rotavirus antigen in the screening test on admission. The patient underwent CBT at 6 months of age (27 weeks 1 day) at the National Center for Child Health and Development. Rotavirus antigen test turned negative 32 days after CBT.

The third case (P3) was that of a patient with X-SCID who was vaccinated with RV5 at 9 weeks of age (4). Three vaccine doses were administered; however, the patient was admitted to 


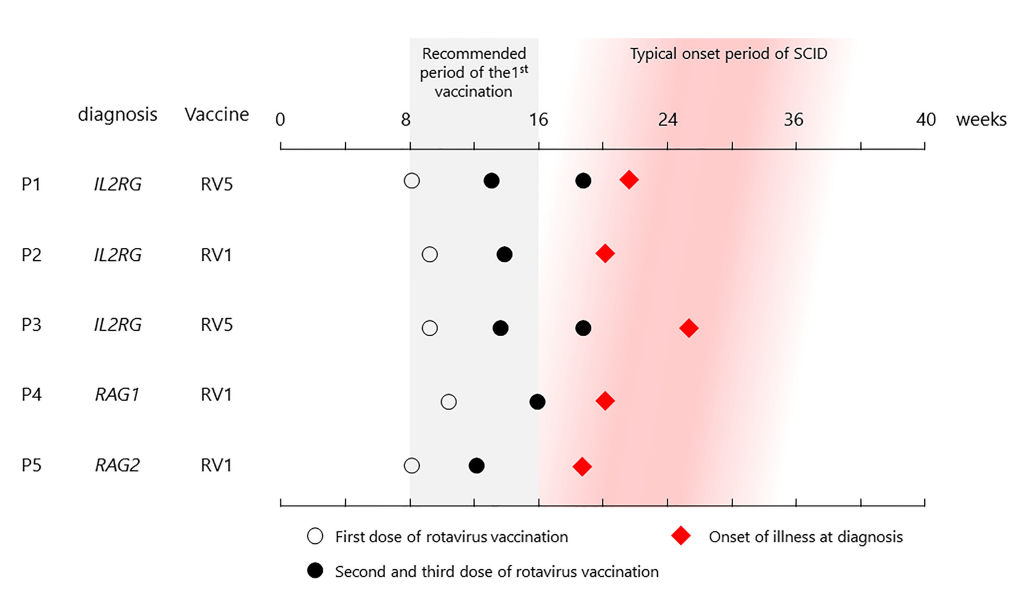

FIGURE 1 | List of vaccination and onset dates in patients. All patients received their first vaccine dose within the recommended period, and each showed symptoms at the typical onset time.

the hospital because of diarrhea and weight loss ( $\max -15 \%)$ at 5 months. He also had agammaglobulinemia, a lack of T cells and CD132, and was diagnosed with X-SCID (IL2RG: c.74_75insAC, p.T26Rfs). The patient underwent CBT at 9 months of age (42 weeks 6 days) at Hokkaido University; however, he remained positive for rotavirus antigen until then. Rotavirus antigen turned negative 27 days after CBT, and steady body weight gain was achieved since then.

The fourth case (P4) was that of a boy with recombination activating gene 1 (RAG1) deficiency, an autosomal recessive SCID, and was vaccinated with RV1 at 10 weeks of age. He was initially admitted to the Kyushu University Hospital at 4 months of age with rhinovirus infection and interstitial pneumonia and was then diagnosed with RAG1 deficiency (RAG1: c.2209C>T, p.R737C and c.2923C>T, p.R975W). In addition, he had loose stools and was positive for the rotavirus antigen. He underwent bone marrow transplantation (BMT) from HLA-matched sibling at 6 months of age (26 weeks 0 days). Rotavirus antigen test turned negative 15 days after BMT.

The fifth case (P5) was that of a girl with autosomal recessive SCID, RAG2 deficiency (RAG2: c.143T $>$ A, p.L48Q and c.419A $>$ G, p.H140R) who developed PCP at 4 months of age after receiving RV1 (9). During the course of treatment at Nagoya University Hospital for disease management, vomiting and increased gastric remnants were observed, and she was diagnosed as having rotavirus antigen-positive gastroenteritis. Thus, the first CBT was performed at 8 months of age without conditioning treatment because of severe PCP. Although she had mix chimerism, rotavirus antigen test turned negative 38 days after the first CBT. The second CBT with conditioning regimen was performed at 17 months, and she achieved full chimerism and revolved PCP completely.

In all cases, each patient completed the default doses of RV1 or RV5. One case (P2) did not demonstrate GI symptom, and rotavirus antigen was incidentally identified by screening on admission. Two cases (P1 and P4) had minor symptoms with loose stools, and one (P5) had mild symptoms with vomiting. They had no weight loss due to GI symptoms. In contrast, one case (P3) presented with severe diarrhea and weight loss. Total parenteral nutrition was not required in any of them.

\section{Detection of Vaccine-Derived Rotavirus}

Further examination of the rapid test-positive feces revealed that the rotavirus strain from P1 stool was the vaccine-derived strain

TABLE 1 | Clinical features of 5 cases.

\begin{tabular}{|c|c|c|c|c|c|}
\hline & P1 & P2 & P3 & P4 & P5 \\
\hline Gene & IL2RG & IL2RG & IL2RG & RAG1 & RAG2 \\
\hline \multirow[t]{4}{*}{ Variants } & c. $676 \mathrm{C}>\mathrm{T}$ & c.202 G>T & c.74_75insAC & c.2209C>T & c. $143 T>A$ \\
\hline & p.R226C & p.E68* & p.T26Rfs & p.R737C & p.L48Q \\
\hline & & & & c.2923C>T & c. $419 A>G$ \\
\hline & & & & p.R975W & p.H140R \\
\hline Vaccine & RV5 & $\mathrm{RV} 1$ & RV5 & $\mathrm{RV} 1$ & RV1 \\
\hline Total dose & 3 & 2 & 3 & 2 & 2 \\
\hline Severity of Gl symptoms & Mild & None & Severe & Mild & Mild \\
\hline Post HCT days when antigen test turned negative & Day 14 & Day 32 & Day 27 & Day 15 & Day 38 \\
\hline Weight loss due to Gl symptoms & None & None & Severe & None & None \\
\hline TPN & No & No & No & No & No \\
\hline
\end{tabular}

RV1, Rotarix ${ }^{\circledR}$; RV5, RotaTeq ${ }^{\circledR}$; Gl, gastrointestinal; HCT, hematopoietic cell transplantation; TPN, total parenteral nutrition. 
by Sanger sequence, and those from the others were vaccine strains derived by RT-PCR. Sequential data of viral loads in stools were demonstrated in P2, P3, and P4, and they decreased after HCT (Figure 2). In addition, the vaccine strain was detected in the serum of $\mathrm{P} 2$ and $\mathrm{P} 4$, suggesting that the disease developed into a systemic infection.

\section{DISCUSSION}

Patients with SCID are asymptomatic until 3-4 months of age when maternal IgG levels decrease, making it challenging to diagnose SCID based on physical examination and history alone without knowing family history (1). The recommended age for the first dose of rotavirus vaccine is $8-15$ weeks, considering when the infection becomes severe; late administration should be avoided (10). The five SCID cases from Japan suggest that rotaviruses are continuously excreted from the intestinal tract of infected patients, even if they do not show severe gastroenteritis symptoms. Although transmission of the vaccine strain rotavirus is not considered a major problem in healthy infants (11), nosocomial infection thought to be derived from the excreted vaccine strain of rotavirus was observed in P5 (9). Therefore, live rotavirus vaccines should not be administered before diagnosing patients with SCID, even if the disease is not severe. With an annual incidence of approximately 1 in 50,000, SCID is not a rare disease compared with other diseases subjected to mass screening of newborns and given the existence of curative treatments, such as HCT and gene therapy, thereby making it appropriate for screening (12) (unfortunately, gene therapy is not available in Japan). Early diagnosis is necessary to exclude patients with SCID from live rotavirus vaccination targets. T-cell receptor excision circles (TRECs) quantitative PCR assay using neonatal dried blood spots is a useful diagnostic method (13).

Furthermore, a recent review of newborn screening with TRECs showed that the sensitivity of the test for SCID is $100 \%$, demonstrating its usefulness (14). In 2018, TRECs screening for newborns was implemented in all states of the United States, spreading globally, including Europe. However, regional disparities in medical care is a problem because only a few regions in Japan have implemented this system. Assuming that 850,000 babies are born annually in Japan, approximately 15-20 per year will be diagnosed with SCID. Therefore, it is desirable to establish a screening system of TRECs for all newborns in Japan at an early stage.

\section{CONCLUDING REMARK}

We described five SCID cases associated with rotavirus-derived infection in Japan with some showing severe gastroenteritis. Therefore, we should introduce TRECs screening for newborns to improve prognosis of SCID in Japan.
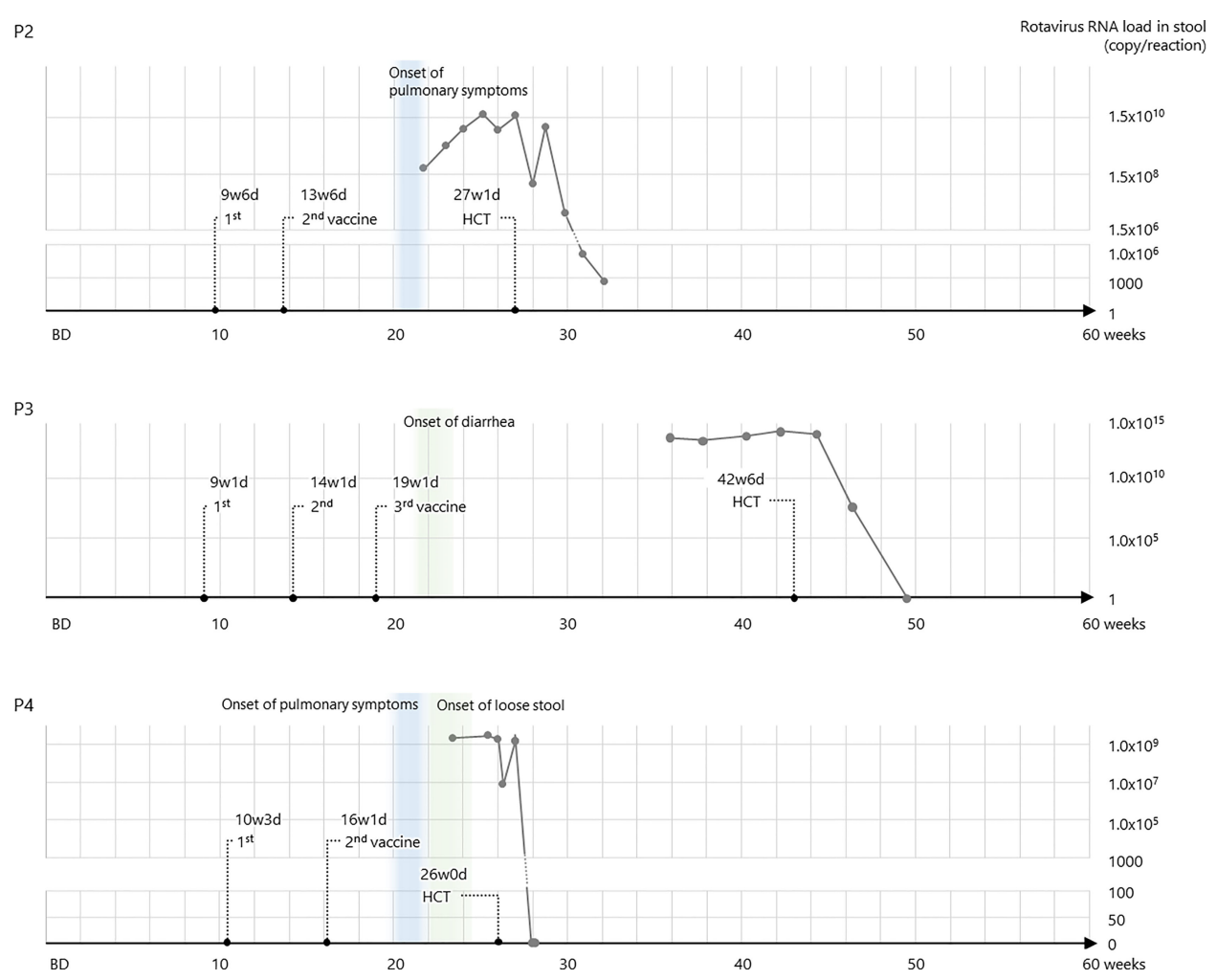

FIGURE 2 | RV5 genotype G1 RNA loads in the stool samples of patients 2, 3, and 4. 


\section{DATA AVAILABILITY STATEMENT}

The raw data supporting the conclusions of this article will be made available by the authors, without undue reservation.

\section{ETHICS STATEMENT}

The studies involving human participants were reviewed and approved by the ethics boards of the Tokyo Medical and Dental University and Fujita Health University School of Medicine. Written informed consent to participate in this study was provided by the participants' legal guardian/next of kin.

\section{AUTHOR CONTRIBUTIONS}

KT and YK wrote the manuscript. YK, HirM, and NM performed genetic analysis. TT, KeI, AI, MY, TaY, HidM, NT,

\section{REFERENCES}

1. Tasher D, Dalal I. The Genetic Basis of Severe Combined Immunodeficiency and its Variants. Appl Clin Genet (2012) 5:67-80. doi: 10.2147/TACG.S18693

2. Patel NC, Hertel PM, Estes MK, de la Morena M, Petru AM, Noroski LM, et al. Vaccine-Acquired Rotavirus in Infants With Severe Combined Immunodeficiency. N Engl J Med (2010) 362:314-9. doi: 10.1056/ NEJMoa0904485

3. Bogaert D, Van Schil K, Taghon T, Bordon V, Bonroy C, Dullaers M, et al. Persistent Rotavirus Diarrhea Post-Transplant in a Novel JAK3-SCID Patient After Vaccination. Pediatr Allergy Immunol (2016) 27:93-6. doi: 10.1111/ pai. 12455

4. Yoshikawa T, Ihira M, Higashimoto Y, Hattori F, Miura H, Sugata K, et al. Persistent Systemic Rotavirus Vaccine Infection in a Child With X-Linked Severe Combined Immunodeficiency. J Med Virol (2019) 91(6):1008-13. doi: 10.1002/jmv.25410

5. Tomoda T, Mitsuiki N, Okano T, Tanaka-Kubota M, Miyamoto S, Kimura S, et al. A Case of Severe Combined Immunodeficiency With Vaccine-Acquired Rotavirus. J Japan Pediatr Soc (2016) 120:1643-8.

6. Gautam R, Esona MD, Mijatovic-Rustempasic S, Ian Tam K, Gentsch JR, Bowen MD. Real-Time RT-PCR Assays to Differentiate Wild-Type Group A Rotavirus Strains From Rotarix $\left({ }^{\circledR}\right)$ and RotaTeq $\left({ }^{\circledR}\right)$ Vaccine Strains in Stool Samples. Hum Vaccin Immunother (2014) 10:767-77. doi: 10.4161/hv.27388

7. Mijatovic-Rustempasic S, Tam KI, Kerin TK, Lewis JM, Gautam R, Quaye O, et al. Sensitive and Specific Quantitative Detection of Rotavirus A by OneStep Real-Time Reverse Transcription-PCR Assay Without Antecedent Double-Stranded-RNA Denaturation. J Clin Microbiol (2013) 51:3047-54. doi: 10.1128/JCM.01192-13

8. Okano T, Nishikawa T, Watanabe E, Watanabe T, Takashima T, Yeh TW, et al. Maternal T and B Cell Engraftment in Two Cases of X-Linked Severe Combined Immunodeficiency With IgG1 Gammopathy. Clin Immunol (2017) 183:112-20. doi: 10.1016/j.clim.2017.08.003

9. Miura H, Taniguchi K, Narita K, Kawamura Y, Kozawa K, Muramatsu H, et al. Nosocomial Infection With Rotavirus Vaccine Strain in Paediatric Patients With Immunodeficiency. J Hosp Infect (2021) S0195-6701:00440-0. doi: 10.1016/j.jhin.2021.12.009
TosM, MK, KE, and MI provided clinical information. SO, KoI, and TomM provided critical discussion. TeY revised the manuscript. HK conceptualized the study and revised the manuscript. All authors contributed to the article and approved the submitted version.

\section{FUNDING}

This study was partly funded by Takeda Science Foundation.

\section{ACKNOWLEDGMENTS}

We thank the patients and their parents for participating in this study. We would like to thank Enago (www.enago.jp) for English language review.

10. Vesikari T, Van Damme P, Giaquinto C, Dagan R, Guarino A, Szajewska H, et al. European Society for Paediatric Infectious Diseases Consensus Recommendations for Rotavirus Vaccination in Europe: Update 2014 Pediatr Infect Dis J (2015) 34:635-43. doi: 10.1097/INF.0000000000000683

11. Hiramatsu H, Suzuki R, Nagatani A, Boda H, Miyata M, Hattori F, et al. Rotavirus Vaccination can be Performed Without Viral Dissemination in the Neonatal Intensive Care Unit. J Infect Dis (2018) 217:589-96. doi: 10.1093/infdis/jix590

12. Wilson JMG, Jungner G. (1968). Geneva: WHO. Available at: http://www. who.int/bulletin/volumes/86/4/07-050112BP.pdf.

13. van der Spek J, Groenwold RH, van der Burg M, van Montfrans JM. TREC Based Newborn Screening for Severe Combined Immunodeficiency Disease: A Systematic Review. J Clin Immunol (2015) 35:416-30. doi: 10.1007/s10875015-0152-6

14. Morinishi Y, Imai K, Nakagawa N, Sato H, Horiuchi K, Ohtsuka Y, et al. Identification of Severe Combined Immunodeficiency by T-Cell Receptor Excision Circles Quantification Using Neonatal Guthrie Cards. J Pediatr (2009) 155:829-33. doi: 10.1016/j.jpeds.2009.05.026

Conflict of Interest: The authors declare that the research was conducted in the absence of any commercial or financial relationships that could be construed as a potential conflict of interest.

Publisher's Note: All claims expressed in this article are solely those of the authors and do not necessarily represent those of their affiliated organizations, or those of the publisher, the editors and the reviewers. Any product that may be evaluated in this article, or claim that may be made by its manufacturer, is not guaranteed or endorsed by the publisher.

Copyright (c) 2022 Tanita, Kawamura, Miura, Mitsuiki, Tomoda, Inoue, Iguchi, Yamada, Yoshida, Muramatsu, Tada, Matsui, Kato, Eguchi, Ishimura, Ohga, Imai, Morio, Yoshikawa and Kanegane. This is an open-access article distributed under the terms of the Creative Commons Attribution License (CC BY). The use, distribution or reproduction in other forums is permitted, provided the original author(s) and the copyright owner(s) are credited and that the original publication in this journal is cited, in accordance with accepted academic practice. No use, distribution or reproduction is permitted which does not comply with these terms. 